\title{
The Effect of Ossicular Chain Disorders on Sound Transmission and Tinnitus Perception using Electrical Model
}

\author{
Manal Riad ${ }^{1}$, Omar Bouattane ${ }^{2}$ \\ ${ }^{1,2}$ SSDIA Laboratory, ENSET-M, Hassan II University of Casablanca, Mohammedia, Morocco \\ m.riadmanal@gmail.com, o.bouattane@gmail.com
}

\begin{abstract}
Human middle ear ensures sound transfer due its ossicular chain, any disorder or abnormalities in this structure leads to a conductive hearing loss (CHL). Tinnitus is a health problem, associated with hearing loss, it remains a devastating symptom. In this work, we present an electrical model of the human middle ear including middle ear cavities (ZMEC), tympanic membrane with ossicular chain (Zтос), and stapes complex with cochlea load (Zsc). This model is modified to represent more closely the related pathologies affecting the middle ear. We will focus our analysis on ossicular chain disorder by studying the effect of increasing ossicular chain (OC) stiffness and mass in both normal middle ear structures and disconnected stapes superstructure. The change in middle ear structures and impedance allows us to simulate ossicular chain disorder effects and analyze their impact on sound transmission. This analysis allowed us to know if this disorder can eventually cause tinnitus. The results showed that the effect of ossicular chain anomalies can be studied based on frequency response of middle ear transfer function by applying only the principle of mass and stiffness, and demonstrate compared to clinical results the efficiency and simplicity of using the electrical model.
\end{abstract}

Key words: Ossicular chain disorder, human Middle ear, Electrical model, sound transmission, Tinnitus.

\section{INTRODUCTION}

Tinnitus is a complex symptom of failing hearing, defined as a perception of noise or buzzing in the ear [1]. For several decades tinnitus studies have been focused essentially on the cochlea [2], unfortunately, the process of hearing is not limited to the state of the cochlear organ in the inner ear and this is due to the mechanism of sound transmission throughout the auditory system, the efficiency with which the acoustic energy is transmitted throughout this system is essential for uninterrupted hearing process.

The human middle ear ensured sound transmission throughout the auditory system. Sound collected in the external ear is transformed into mechanical vibrations of the tympanic membrane and the ossicular chain (Malleus, incus and, stapes) and then into a traveling wave in the cochlea fluid. To adapt the low acoustic impedance of the ear and the high impedance of the cochlea fluid, the middle ear function as an impedance matching unit [3], and this is due to the lever mechanism of the ossicular chain. Like any mechanical system, its functioning depends strongly on the mass of ossicles, eardrum, and stiffness of membrane and ligaments, any disorder in the ossicular chain will reduce the efficiency of sound transmission.

For better understanding the mechanical behavior of the middle ear, and to predict its normal and pathological mechanism, different models have been developed in the literature using different approaches as reviewed in [4], one of this approaches uses circuits and finite element models, having each one, its advantage and disadvantage, electrical circuit models have generally problem to represent the complexity of real middle ear structure [5] as complex vibration of the tympanic membrane [6], [7] or three-dimensional motion of the ossicular chain at high frequencies [8].

Despite these limitations, compared to the finite element model, the simplicity of the electrical circuit model allows us to provide insight into the mechanic of the middle ear which helps it to represent its band-pass characteristic of the transfer function and to analyze the effect of each component with a reduced computational cost. Based on middle ear impedance the early analog electro-acoustical model is developed to represent in a quantitative way the different structure of the middle ear [9], several other models are based on this electrical model in [10], [11] and was adapted to represent the human middle ear nonlinearities in [12].

The aims of this paper are: (1) analyze the frequency response of increased ossicular chain mass and stiffness with intact tympanic membrane for a normal middle ear structures and disconnected middle ear stapes superstructure. (2) Study the effect of these disorders on sound transmission and tinnitus perception. The paper is structured as follows: the model and its substructure are described in section II, in section III we present the modified model of the pathological middle ear and simulate the effect of changing ossicular chain structure and impedance. Finally, the work is concluded in section IV. 


\section{MIDDLE EAR ELECTRICAL MODEL}

The human middle ear network can be subdivided into three impedance blocks [13], this structure is common in a typical network model as shown in Figure 1.

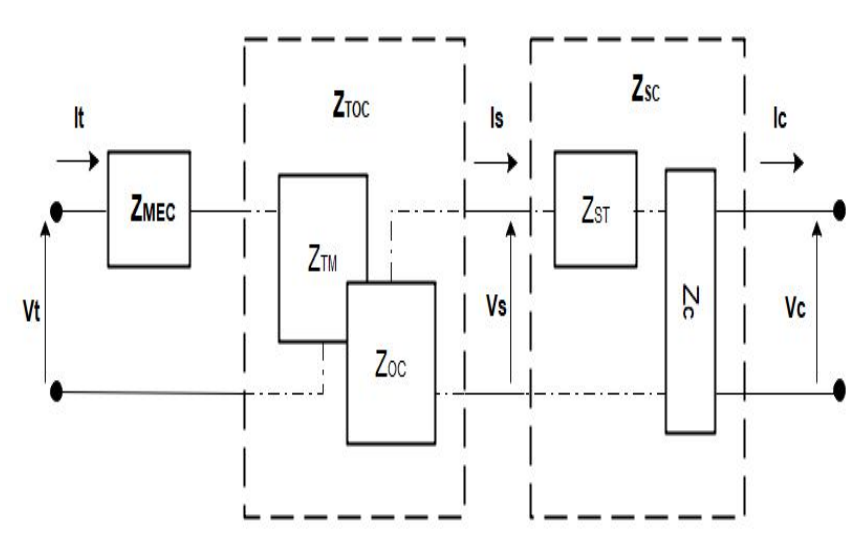

Figure 1 : Middle ear network model adapted from [13]

The first block ZMEC represents the impedance of middle ear cavities decoupled from the mechanical pathway, the second one Zтос represents the impedance of the tympanic membrane (TM) and ossicular chain up to incudo stapedial junction representing two-port networks of a conductive pathway and the last one Zsc represent the stapes complex and cochlea input impedance. The middle ear is considered as a mechanical system, the differential equations of this complex mechanical behavior can be represented using electrical circuits Figure 2 as in [12].

The electrical analog circuit models have been extensively used in auditory system studies, due to their simplicities to represent in a very compact way the middle ear function. Based on electro-acoustical analogy, Voltage and current are analogous respectively to the acoustic pressure and volume velocity, Resistors (R), capacitor (C), and inductor (L) of the given circuit model represent acoustic resistors, compliance, and acoustic mass.

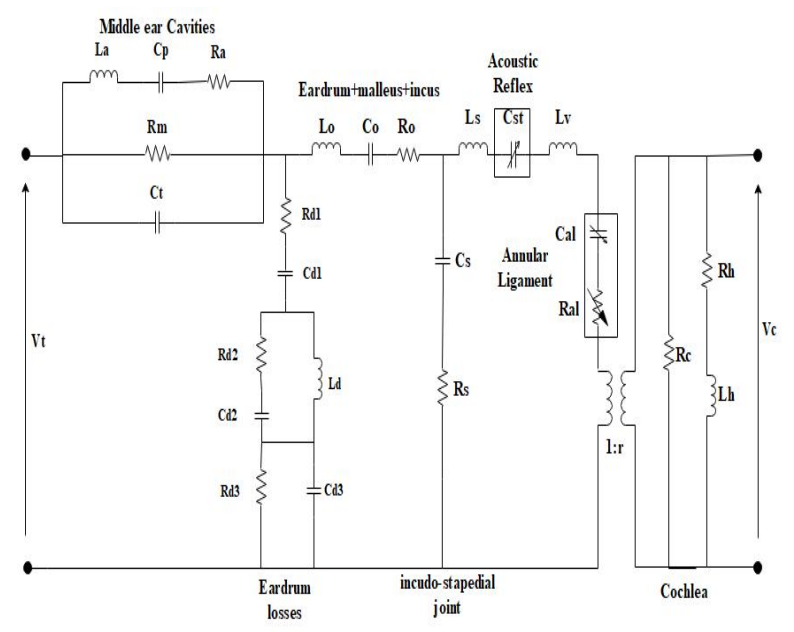

Figure 2 : Electrical Model of human middle ear [12]
The electrical components values are given based on the transfer function and impedance measurements, and anatomical data. Electrical impedance is hence analogous to acoustic impedance. The impedance $(\mathrm{Z})$ of the electrical elements are given by:

$$
\left\{\begin{array}{l}
Z_{R}=R \\
Z_{C}=\frac{1}{j c w} \\
Z_{L}=j L w
\end{array}\right.
$$

The approximate values of Table 1 give the equivalents component of the real ear.

Table 1 : Electrical model components values [12]

\begin{tabular}{|ll|}
\hline Middle ear cavities: & TM and Ossicular chain \\
$\mathrm{La}=12 \mathrm{mH}$ & $\mathrm{Rd} 1=20 \Omega$ \\
$\mathrm{Cp}=3.6 \mu \mathrm{F}$ & $\mathrm{Rd} 2=105 \Omega$ \\
$\mathrm{Ct}=0.35 \mu \mathrm{F}$ & $\mathrm{Rd} 3=12500 \Omega$ \\
$\mathrm{Ra}=20 \Omega$ & $\mathrm{Cd} 1=0.5 \mu \mathrm{F}$ \\
$\mathrm{Rm}=420 \Omega$ & $\mathrm{Cd} 2=0.3 \mu \mathrm{F}$ \\
$\mathrm{Stapes}+\mathrm{Cochlea}:$ & $\mathrm{Cd} 3=0.2 \mu \mathrm{F}$ \\
$\mathrm{Ls}=8 \mathrm{mH}$ & $\mathrm{Ld}=15 \mathrm{mH}$ \\
$\mathrm{Lv}=21 \mathrm{mH}$ & $\mathrm{Lo}=40 \mathrm{mH}$ \\
$\mathrm{Cst}=0.25 \mu \mathrm{F}-\mathrm{infinity}$ & $\mathrm{Ro}=65 \Omega$ \\
$\mathrm{Ral}=200 \Omega-2.6 \mathrm{~K} \Omega$ & $\mathrm{Co}=1.4 \mu \mathrm{F}$ \\
$\mathrm{Cal}=0.05 \mu \mathrm{F}-0.4 \mu \mathrm{F}$ & $\mathrm{Rs}=170 \Omega$ \\
$\mathrm{Rc}=1211 \Omega$ & $\mathrm{Cs}=0.03 \mu \mathrm{F}$ \\
$\mathrm{Rh}=850 \Omega$ & \\
$\mathrm{Lc}=150 \mathrm{mH}$ & \\
\end{tabular}

\subsection{Middle ear substructures description}

\section{A. Middle ear cavities:}

The electrical model of human middle ear cavities of as shown in Figure 2 can be written as:

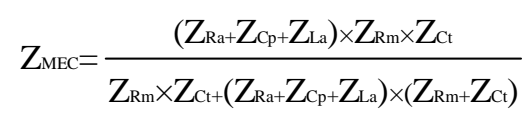

The human middle ear cavities are divided into two air spaces, the first one is directly behind the tympanic membrane with compliance $C_{t}$ and the second with compliance $C p$. The compliance of middle ear cavities depends on the measurements of the equivalent volume of the association of 
the two compliances as shown in $[14,15]$ and computed using Eq. (3)

$$
C=C t+C p=\frac{V}{\rho c^{2}}
$$

With $\mathrm{V}$ and $\rho$ represent the volume and density of air respectively, and $\mathrm{c}$ is the speed of sound. The inductance $L a$ and the resistance $R a$ represent the acoustic mass and acoustic resistance respectively due to the narrow passage between the two parts of cavities and resistance $R m$ represents the sound absorption.

\section{B. Tympanic membrane and ossicular chain}

The mechanic of tympanic membrane and ossicular chain in block Zтос Figure 1 is represented by the electrical model of Figure 2 as $\boldsymbol{\pi}$ network model with three impedances Ztm, Zoc1 and Zoc2 as shown in Figure 3.

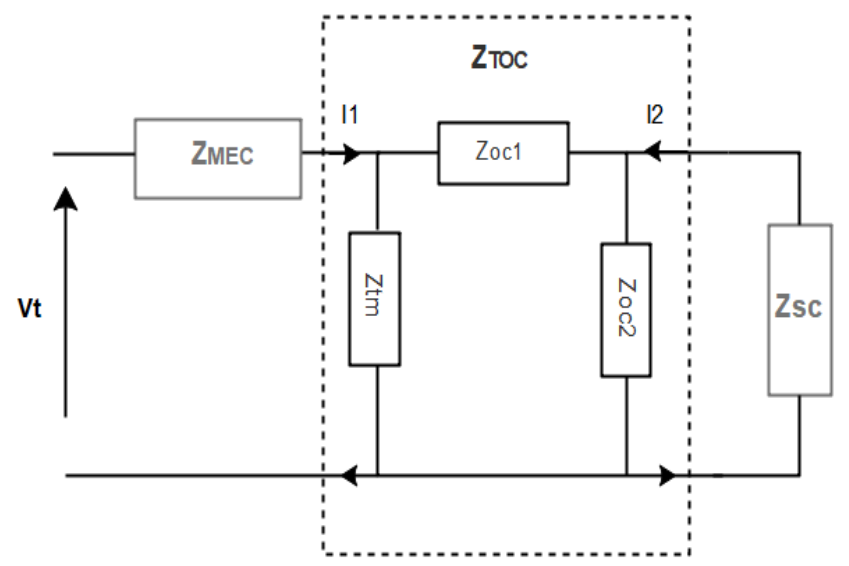

Figure 3 : Two port network ZTOC

The tympanic membrane is modeled as one-dimensional cylinder with characteristic impedance Ztm.

$Z t m=\left[Z_{R d 1}+Z_{C d 1}\right]+\left[\frac{Z_{L d} \times\left(Z_{R d 2}+Z_{C d 2}\right)}{Z_{L d}+Z_{R d 2}+Z_{C d 2}}\right]+\left[\frac{\left(Z_{R d 3} \times Z_{C d 3}\right)}{Z_{R d 3}+Z_{C d 3}}\right]$

At low frequencies, the tympanic membrane is modeled as a rigid mass with the mass of malleus and incus [9] with impedance characteristic Zoc1 expressed as:

$$
Z_{O C 1}=Z_{L o}+Z_{C o}+Z_{R o}
$$

The malleus and incus are considered tightly fused [9], so the flexibility of the incudo-mallear joint is not included in our model. The impedance that represents the incudo-stapedial joint is expressed as:

$$
Z_{O C 2}=Z_{C s}+Z_{R s}
$$

The ossicular chain is modeled from the malleus footplate coupled with eardrum up to the incudo-stapedial joint. However, the stapes is part of the ossicular chain but it will be considered as a separate part due to its motion complexity.

\section{Stapes Complex and cochlea}

The behavior of the human middle ear is linearly in normal hearing but becomes nonlinear when it is exposed to a very loud sound. We have two nonlinearities due to the acoustic reflex and the effect of the annular ligament presented in [12]. These nonlinearities disturb the transfer function of the middle ear especially at low frequencies.

The impedance matching between the tympanic membrane and the cochlea are symbolized using an ideal electrical transformer, the acoustic transformer ratio symbolized (1:r) is defined as follows:

$$
r=\operatorname{Lr} \times A r
$$

Where:

$$
A_{r}=A_{t m} / A_{f p}
$$

Using the anatomical data in Table 2 and equation (7) the transformer ratio is closed to 24,8 . The cochlea load $R c, R h$ and $L h$ viewed from the input side of the transformer are given hereby:

$$
\left\{\begin{array}{l}
R c^{\prime}=R c \times r^{2} \\
R h^{\prime}=R h \times r^{2} \\
L h^{\prime}=L h \times r^{2}
\end{array}\right.
$$

Table 2 : Human middle ear anatomical data [16]

$\begin{array}{lll}\text { Eardrum area } & \text { Atm } & 60 \mathrm{~mm} 2 \\ \text { Footplate area } & \text { Afp } & 3.14 \mathrm{~mm} 2 \\ \text { Lever ratio } & \text { Lr } & 1.3 \\ \text { Area ratio } & \text { Ar } & 19\end{array}$

\subsection{Middle ear transfer function}

The middle ear can be characterized as shown in Figure 4 as two-port system with the first port in the tympanic membrane and the second one in the entrance of cochlea. This two port system can be represented as cascade connection of two-port network representing the tympanic membrane and ossicular chain with two one-port network.

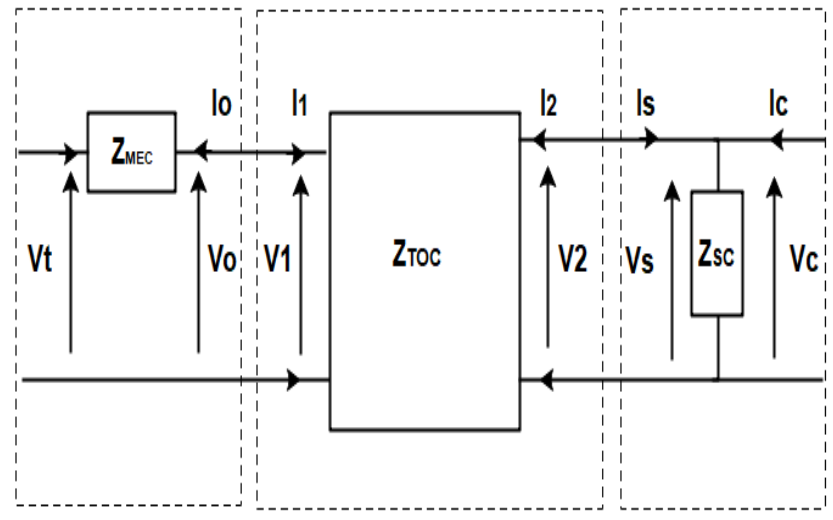

Figure 4 : Comprehensive two-port model adapted from [17] 
The impedance block ZMEC is one-port network representation of the middle ear cavities impedance, the relationship between the input and output of the one-port system can be written as:

$$
\left[\begin{array}{c}
V o \\
I o
\end{array}\right]=\left[\begin{array}{cc}
1 & Z_{M E C} \\
0 & 1
\end{array}\right]\left[\begin{array}{c}
V t \\
-I t
\end{array}\right]
$$

This two-port system can be represented with two-port $\mathrm{ABCD}$-parameters matrix which relates the input and the output of this impedance block and allow for characterization of the tympanic membrane and ossicular chaine independent of the influence of the termination load, the relationship between the two-port networks can be written as:

$$
\left[\begin{array}{c}
V 2 \\
I 2
\end{array}\right]=\left[\begin{array}{ll}
A_{\text {тос }} & \boldsymbol{B}_{\text {тос }} \\
\boldsymbol{C}_{\text {тос }} & D_{\text {тос }}
\end{array}\right]\left[\begin{array}{c}
V 1 \\
-I 1
\end{array}\right]
$$

With

$$
\left\{\begin{array}{l}
A_{T o C}=1+\frac{Z o c 1}{Z t m} \\
B_{T o C}=Z o c 1 \\
C_{T o C}=\frac{Z t m+Z o c 1+Z o c 2}{Z o c 2 \times Z t m} \\
D_{T o C}=\frac{Z o c 1}{Z o c 2}+1
\end{array}\right.
$$

The impedance block Zsc is one-port network representation of the stapes complex and cochlea impedance, the relationship between the input and output of the one-port system can be written as

$$
\left[\begin{array}{l}
V c \\
I c
\end{array}\right]=\left[\begin{array}{cc}
1 & 0 \\
Y_{s c} & 1
\end{array}\right]\left[\begin{array}{c}
V s \\
-I s
\end{array}\right]
$$

With

$$
Y_{S C}=\frac{1}{Z_{S C}}
$$

The relation between the input Vt and output of our system based on [17] can be written as:

$$
\frac{V c}{V t}=\frac{1}{A_{T O C}+\left[C_{T O C} \times Z_{M E C}+\frac{B_{T O C}}{Z_{S C}}+D_{T O C} \times \frac{Z_{M E C}}{Z_{S C}}\right]}
$$

The acoustic pressure at the terminal of the tympanic membrane is analogous to voltage $\mathrm{Vt}$ and the pressure at the cochlea is represented by the voltage Vc. The transfer function of the human middle ear represents the efficiency with which the acoustic energy of sound is transmitted from the tympanic membrane to the cochlea and is expressed:

$$
T(f)=20 \log (V c / V t)
$$

\section{SIMULATING OSSICULAR CHAIN DISORDERS}

The model above Figure 2 and all middle ear models, suppose that the only mechanism of sound transmission is the ossicular chain [18]. This type of transmission is called ossicular coupling. Sound pressure (voltage), volume velocity (current), and acoustic impedance (Impedance Z) represents the variable used to describe the human middle ear system. The description of the relationship between these variables and the structure of the real middle ear helps us to easily identify the phenomenon of sound conduction in the normal and pathological middle ear. A change affecting these middle ear variables or structure leads to a reduction of middle ear transmission, and subsequently a modified transfer function shape as demonstrate in our previous work [19]. The method used in this work is carried out using MATLAB /Simulink software based on the process described in Figure 5, Figure 6 shows the stimulus of our system.

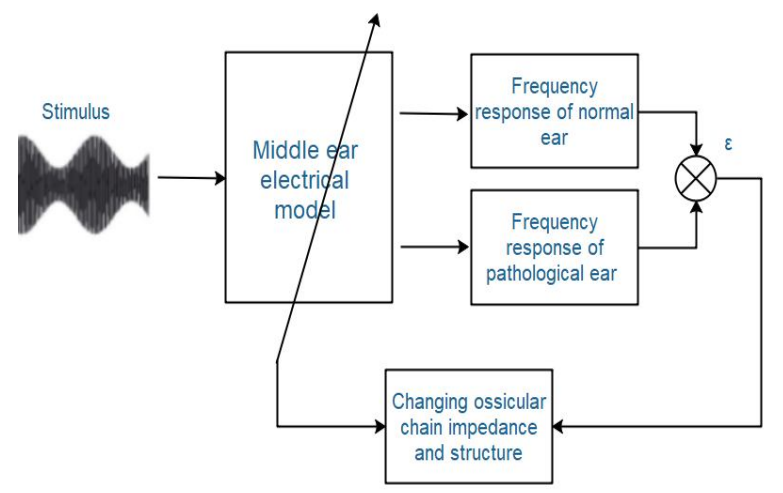

Figure 5: Simulation process of ossicular chain disorder

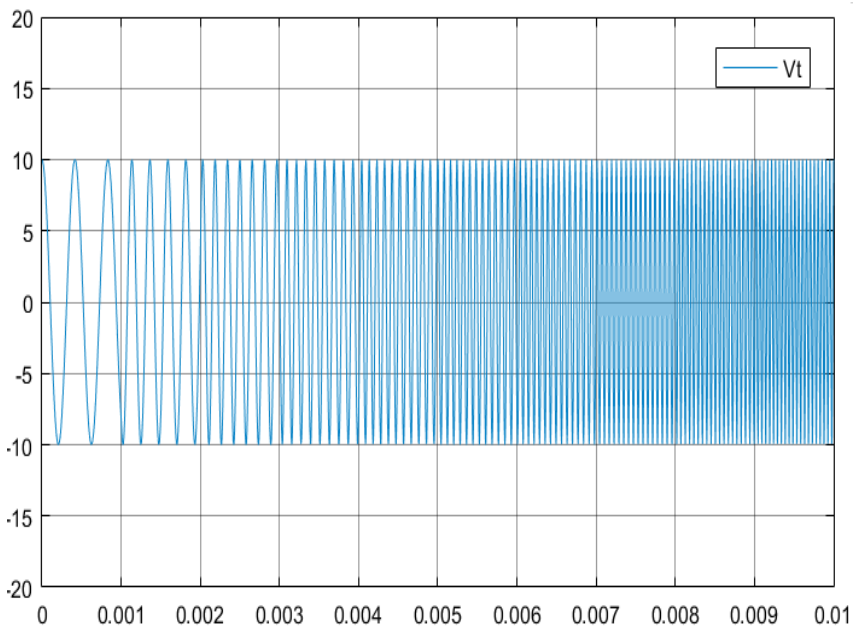

Figure 6: The input signal Vt generated using the Voltage-controlled oscillator VCO

\subsection{Pathological middle ear model}

The circuit model of the human middle ear Figure 1 is modified in order to model the effect of middle ear pathologies including two switches $\mathrm{K} 1$ and $\mathrm{K} 2$ as showns in Figure 7, which are used to modelize the tympanic membrane perforation and complete ossicles disarticulations respectively. 


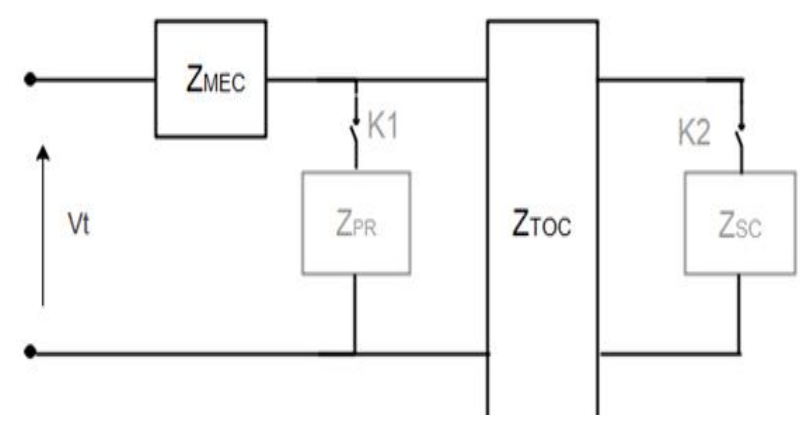

Figure 7: Modified network model to study middle ear pathologies [20][21]

ZPR is the impedance of perforation it depends closely on frequency and perforation diameter, to specify this impedance the perforation is represented as a circular orifice in thin plate [21] is expressed as:

$$
Z_{P R}=\frac{4 \rho 2 \pi f(t+\delta)}{\pi d^{2}} \varphi
$$

Where $\left\{\begin{array}{l}\varphi=-j \frac{J_{o}(k \times d / 2)}{J_{2}(k \times d / 2)} \\ \rho=\frac{8 d}{3 \pi} \text { and } k^{2}=\frac{-j \rho 2 \pi f}{\mu}\end{array}\right.$

The diameter of perforation is represented by $\mathrm{d}$, $\mathrm{f}$ represents frequency, $\rho$ and $\mu$ are the density and viscosity of air. $t$ represents the thickness of the tympanic membrane, it is equal to $0.06 \mathrm{~mm}$ [22]. $J_{0}$ and $J_{2}$ represent the zero and second-order of Bessel function respectively. For studying only ossicular chain disorder we will suppose that the tympanic membrane is not perforated so $\mathrm{K} 1$ will be open in this section.

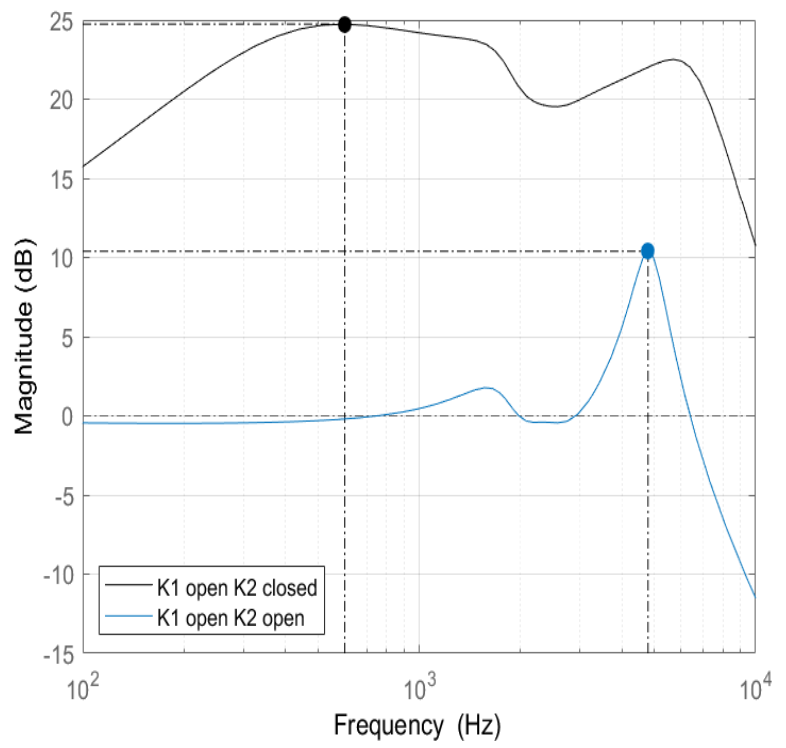

Figure 8: Transfer function of normal middle ear structures and disconnected middle ear stapes superstrure.

\subsection{First state: K1 open, K2 closed}

Several pathologies of the middle ear related to ossicular coupling disorder, which causes hearing loss and other phenomena such as tinnitus, are concerning changing middle ear impedance by changing mass and stiffness [23]. An abnormal increase in mass and stiffness of the ossicles causes a change of the impedance characteristic of these ossicles Zoc. As shown in the first section the ossicular chain is considered from eardrum (TM) up to the incudo-stapedial joint, the tympanic membrane will be considered intact so no change on the impedance characteristic Ztm . The stiffness is the inverse of compliance, so for increased stiffness the compliance is decreased, the acoustic compliance of the ossicular chain is analog to capacities Co and Cs and mass of ossicles is analog to the inductance Lo.

Table 3: Representation of different combination and their corresponding circuit related normal middle ear structures

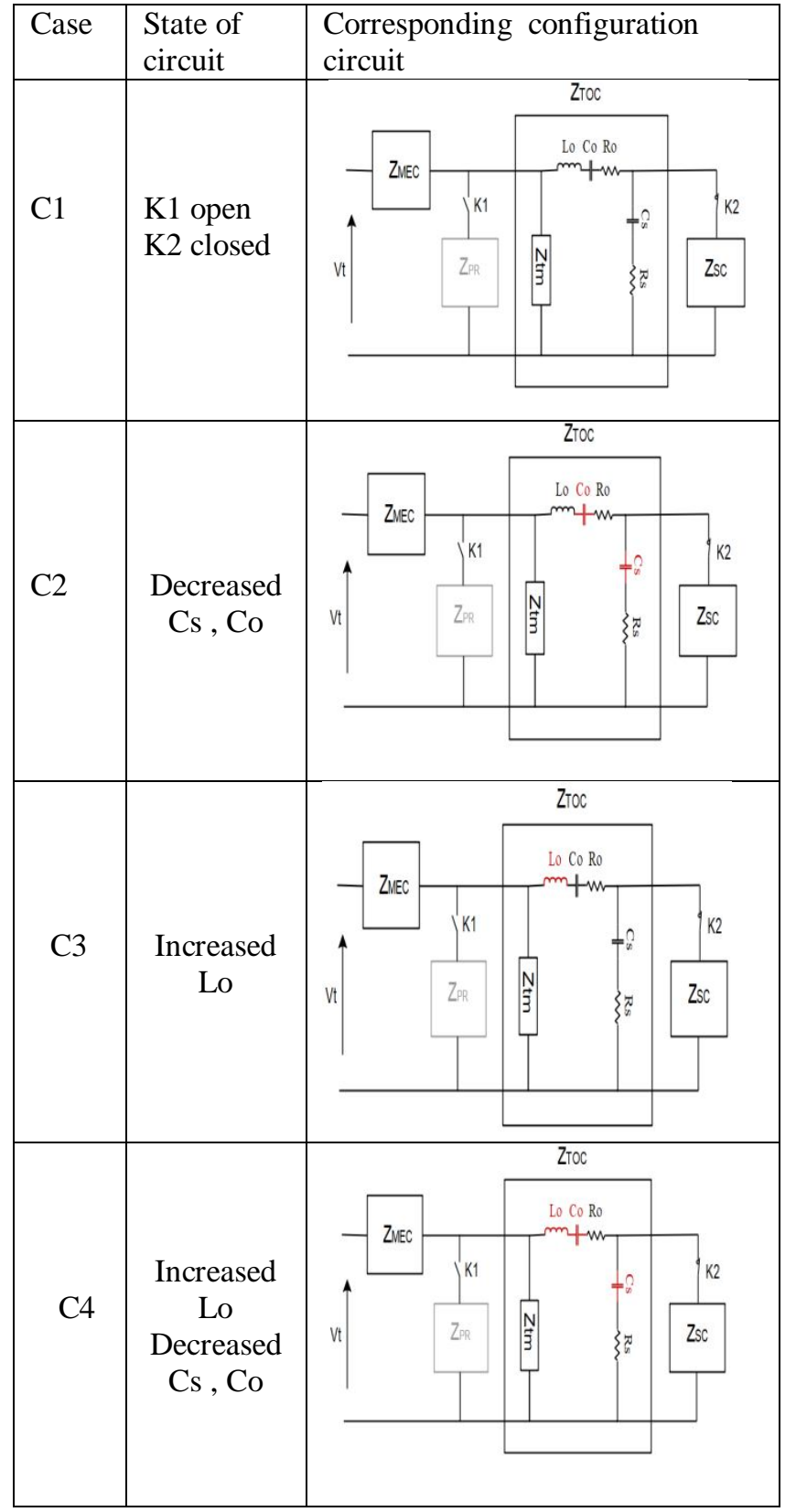




\subsection{Second state: K1 open, K2 open}

Ossicular anomalies include absence or malformation of any ossicles, the majority of abnormalities are associated with other malformation and may involve fixations, defects, or absence of middle ear structures (malleus, incus, stapes) [24] the disconnected stapes it one of these abnormalities modeled in our study by connecting directly the ossicular chain (mallus+incus) to the air space by bypassing the connection to the stapes and cochlea.

Table 4: Representation of different combination and their corresponding circuit related to disconnected stapes superstructure

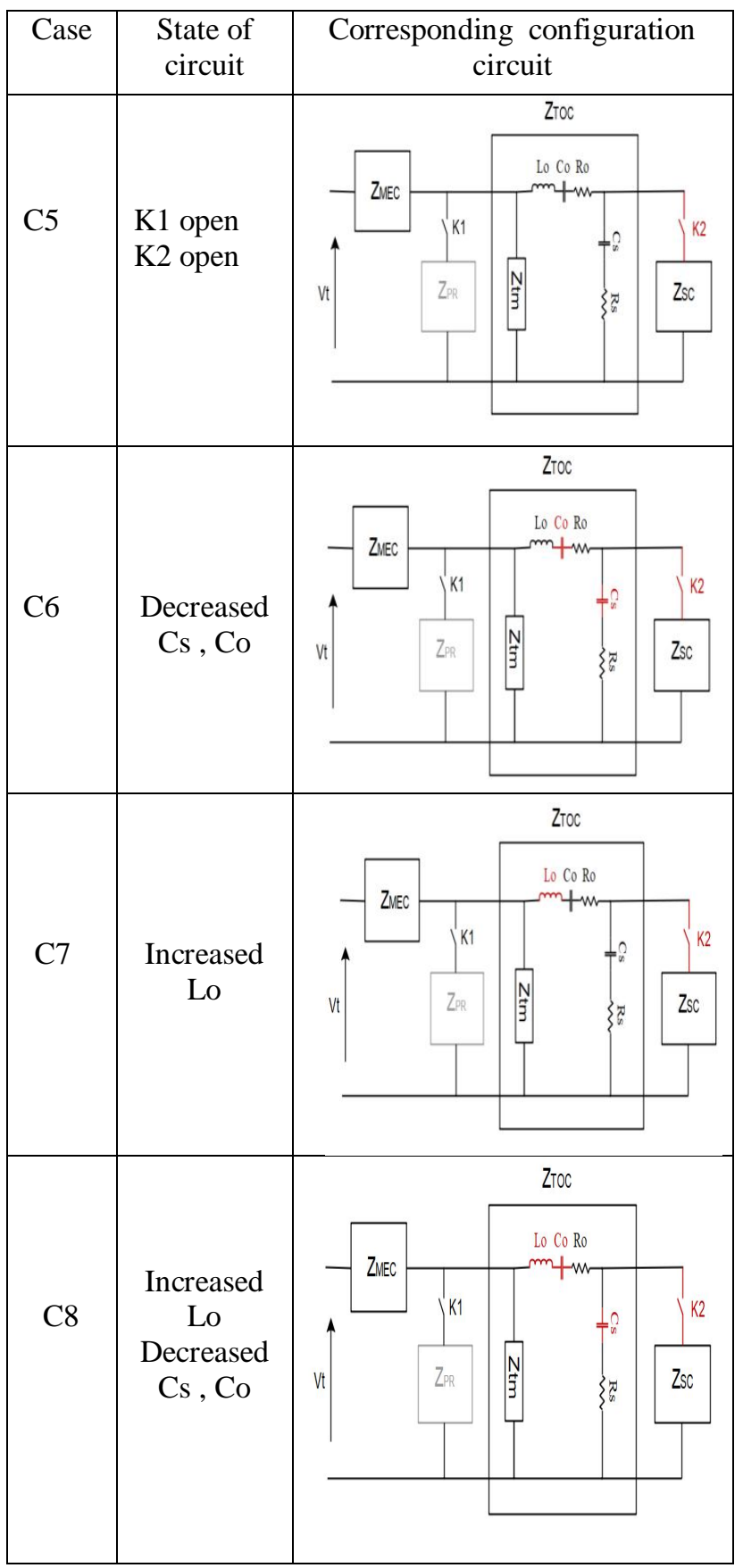

\section{RESULTS AND DISCUSSION}

The effects of increased ossicular chain stiffness and mass were analyzed based on electrical model simulation for both normal middle ear structures and disconnected stapes superstructure. The stiffness and mass will be increased by a factor of 10 than by a factor of 100 , so the values of the capacitors will be reduced by a factor of 0.1 and 0.01 and inductance increased by a factor of 10 than 100

\subsection{Change ossicular chain impedance}

In Figure 8 for normal structures K1 open and K2 closed the sound pressures are $25 \mathrm{Db}$ greater at the cochlea than those at the tympanic membrane, the transfer function of the middle ear represent band-pass characteristic as shown in the figure below across band-pass the pressure gain were different. This transfer function represents a reference of our analysis any change in gain pressure corresponds to a disorder in the middle ear. Table 3 shows the different combination related to the first state representing the change affecting only the ossicular chain impedance.

\section{A. Effect of increasing ossicular chain stiffness or mass}
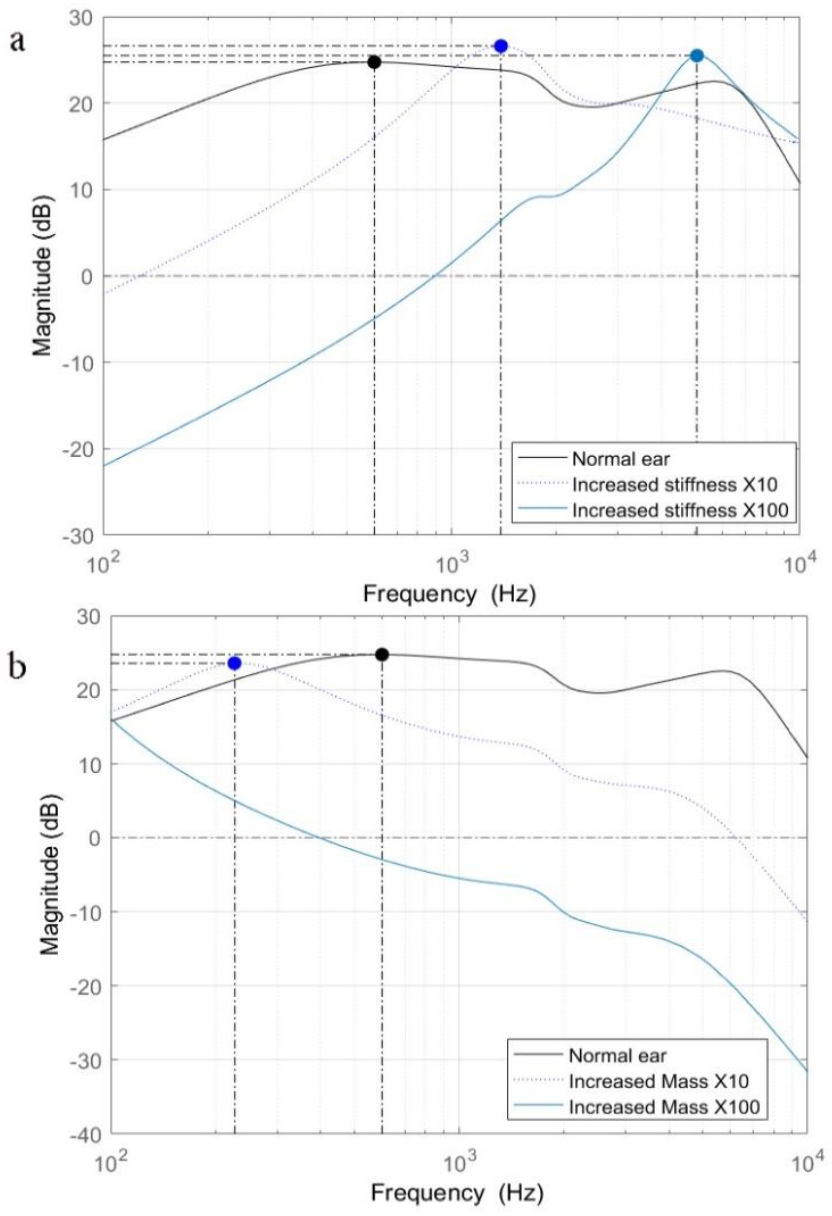

Figure 9: (a) Effect of increased stiffness of ossicular chain on Frequency response of middle ear. The stiffness was increased by factor of 10 and 100. (b) Effect of increased Mass of ossicular chain on Frequency response of middle ear. The mass was increased by a factor of 10 and 100. 
As can be seen in Figure 9.a the gain pressure of the middle ear changes in response to ossicular chain stiffness change. Increased stiffness resulted in reduced pressure gain of middle ear frequency response at low frequencies $<1 \mathrm{kHz}$, and transmit the maximum middle ear transfer function peak response of $26 \mathrm{~dB}$ at $2 \mathrm{KHz}$ for increased stiffness by a factor of 10 and $25 \mathrm{~dB}$ at $6 \mathrm{kHz}$ for increased stiffness by a factor of 100. In Figure 9.b the gain pressure of the middle ear changes in response to ossicular chain mass change. Increased mass resulted in reduced pressure gain of the middle ear frequency response at high frequencies $>1 \mathrm{kHz}$, and transmit the maximum middle ear transfer function peak response of 24 $\mathrm{dB}$ at $300 \mathrm{~Hz}$ for increased mass by a factor of 10 .

\section{B. Effect of increasing both ossicular chain stiffness and mass}

Increasing both Mass and stiffness at the same time as shown in Figure 10 reduces the gain pressure for low and high frequencies, the band-pass characteristic of the middle ear is reduced, Mass help low frequencies to be transmitted and disturbs the transmission of high frequencies and stiffness helps high frequencies and disturb low frequencies, the maximum middle ear transfer function peak response of 25 $\mathrm{dB}$ at $700 \mathrm{~Hz}$ it can be perceived as tinnitus.

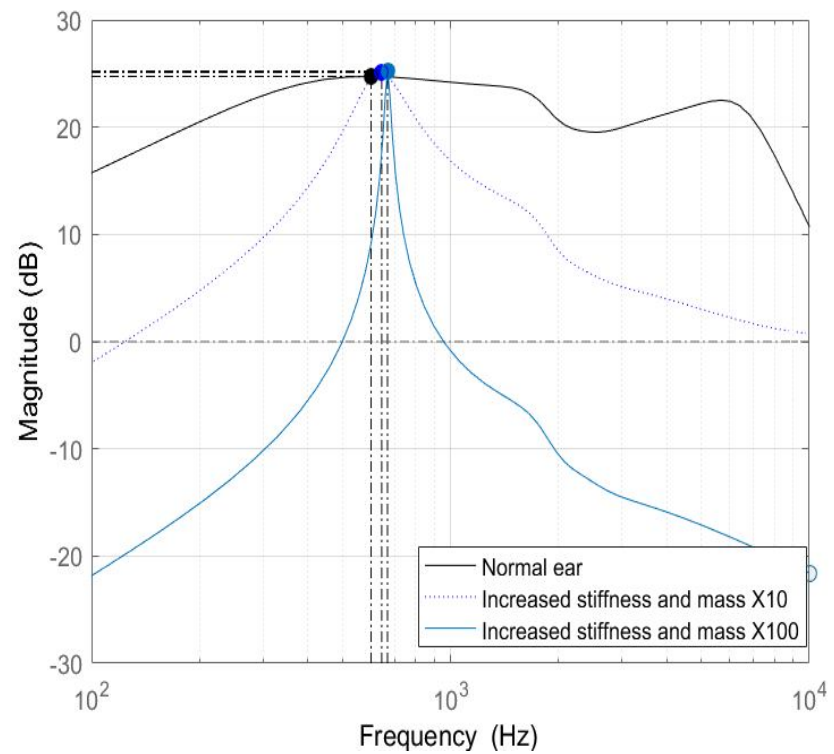

Figure 10: Effect of increased stiffness and mass of ossicular chain on Frequency response of middle ear. The stiffness and mass were both increased by a factor of 10 and 100 .

\subsection{Change ossicular chain structure and impedance}

In presence of an intact tympanic membrane, the disconnected stapes superstructure is represented using Figure 7 by opening $\mathrm{K} 1$ and K2. In Figure 8, at the low frequencies $(\mathrm{f}<1 \mathrm{kHz})$ the gain pressure is $0 \mathrm{~dB}, 25 \mathrm{~dB}$ lower than the gain of a normal ear, for high frequencies $(\mathrm{f}>1 \mathrm{kHz}$ ) the gain increase at $2 \mathrm{kHz}$ until reaches $10 \mathrm{~dB}$ at $5 \mathrm{kHz}$ and decreases for (f $>5 \mathrm{kHz}$ ). This transfer function represents a reference of our analysis to study the effect of combined anomalies and analyses the effect of increased stiffness and mass on disconnected middle ear structures. Table 4 shows the different combination related to the second state representing the change affecting the ossicular chain structure and impedance.

\section{A. Effect of increasing ossicular chain stiffness or mass combined to disconnected stapes superstructure}
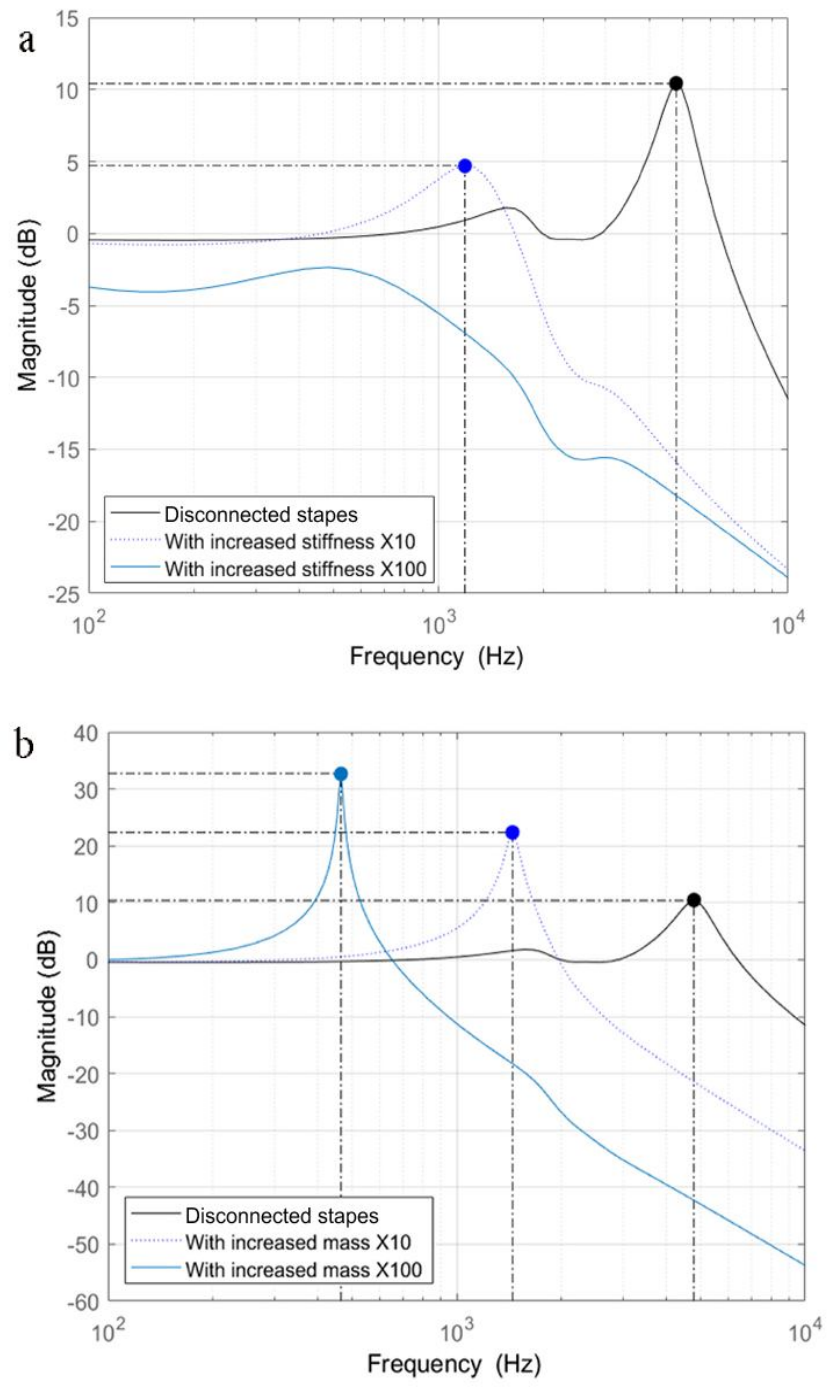

Figure 11: (a) Effect of increased stiffness of ossicular chain on Frequency response of middle ear. The stiffness was increased by factor of 10 and 100. (b) Effect of increased Mass of ossicular chain on Frequency response of middle ear. The mass was increased by a factor of 10 and 100 .

Figure 11 shows the frequency response of middle ear with disconnected stapes superstructure combined with other anomalies of ossicular chain represented by increased mass and stiffness, for increased stiffness Figure 11.a by factor of 10 the peak response decreased from $10 \mathrm{~dB}$ at $5 \mathrm{kHz}$ for only stapes disconnection to $5 \mathrm{~dB}$ at nearly $1 \mathrm{kHz}$, the gain pressure was decreased for all frequencies for increased stiffness by a factor of 100 our system is highly dumped. In Figure 11.b we see for increased mass by a factor o 10 the gain the gain pressure increase from $10 \mathrm{~dB}$ at5 $\mathrm{Khz}$ to $22 \mathrm{~dB}$ at $1,5 \mathrm{kHz}$ and to $32 \mathrm{~dB}$ at nearly $500 \mathrm{~Hz}$ for increased mass by factor of 100 . Combined increased mass with disconnected stapes superstructure can generate oscillation which can be perceived as tinnitus. 


\section{B. Effect of increasing both ossicular chain stiffness and mass combined to disconnected stapes superstructure}

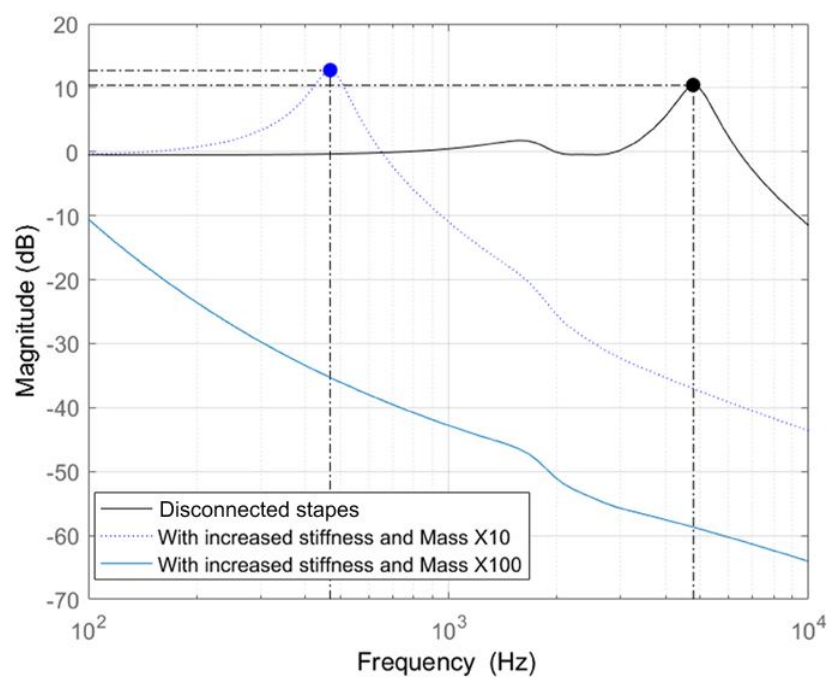

Figure 12: Effect of increased stiffness and mass of ossicular chain on Frequency response of middle ear. The stiffness and mass were both increased by a factor of 10 and 100 .

In Figure 12 for increased both mass and stiffness by factor of 10 the peak response increase from $10 \mathrm{~dB}$ at $5 \mathrm{kHz}$ for only stapes disconnection to $13 \mathrm{~dB}$ at nearly $500 \mathrm{~Hz}$, the gain pressure was decreased for all frequencies for increased stiffness and mass by a factor of 100 our system is highly dumped.

Several studies have been done on human middle ear disorder based on different modeling approaches. Recently the finite elements model is one of the most models used to study and analyze the sound transfer function in normal and pathological conditions. In [25] the finite element model has been used to simulate and study three middle ear disorders: otits media, otosclerosis, and ossicular chain disarticulation based on energy absorbance. in [26] Pathological conditions are analysed to determine the gain or loss in hearing capacity for Cholesteatoma, Partial mallear fixation and variations of material properties in Tympanic membrane (TM) by examining the harmonic response at the stapes footplate, even if they show interesting results, their disadvantage is in terms of the number of used parameters, and their complexity, which can prevent the understanding of fundamental principles in the middle ear in normal and pathological state. For this reason, we chose in our study to use the electrical model not only for its simplicity to describe the behavior of the human middle ear but also for using only two components the capacitor and the inductance, which are analogous respectively to the most significant parameters in the study of pathological conditions: stiffness and mass. Clinical studies shows that the most of middle ear pathologies result an abnormal increase of mass and stiffness which is affect significantly the middle ear transfer function [27]. These clinical results were highly correlated to our electrical model simulation results, which support our choice of using the electrical model to investigate the tinnitus sources that are related to conductive hearing loss.

\section{CONCLUSION}

The purpose of human middle ear modeling is to simulate the middle ear structure and transfer function from the ear canal through ossicles to the cochlea. The human electrical model of this work was used to simulate ossicular chain disorder, it has been suggested in this study that the ossicular chain is the only mechanism of sound transmission in the middle ear.

Ossicular disarticulation with an intact tympanic membrane is a disorder affecting middle ear structure by disconnecting stapes and cochlea from the ossicular chain (Malleus and incus), thus interrupts sound transmission and represents a conductive hearing loss. The sound absorbed by the tympanic membrane is dissipated within the middle ear cavities, the peak response $10 \mathrm{~dB}$ at $5 \mathrm{kHz}$ can be caused by the vibration of air in the cavities of the middle ear, and it can be heard as buzzing in the ear at high frequencies. This proves that the ossicular chain is the main mechanism of sound transmission but not the only.

Mass and stiffness of the ossicular chain affect the particular characteristic of human middle ear, ossicular chain stiffness helps vibrations of high frequencies and disturbs the transmission of low frequencies, while mass disturbs the vibration of high frequencies and helps the transmission of low frequencies. This helps to explain the band-pass of the human middle ear transfer function in normal middle ear structures. Increased mass and stiffness of the ossicular chain represent a disorder affecting the middle ear impedance values, it reduces the band-pass characteristic. This work use the principle of increased mass and stiffness for both normal ossicular chain structure and disconnected structures

As a perspective of this work, we aim to predict how middle ear function is affected by various middle ear pathologies by investigating the effect of each middle ear substructure disorder. The proposed electrical model will allow us to analyze easily the effect of these disorders on sound transmission based principally on middle ear frequency response.

\section{ACKNOWLEDGEMENT}

The authors wish to acknowledge and thank the national center for scientific and technical research (CNRST) for its support during the period of this research.

\section{REFERENCES}

1. L. E. Roberts, J.J. Eggermont, D.M. Caspary, S.E. Shore, J.R. Melcher, and J.A. Kaltenbach. Ringing ears: the neuroscience of tinnitus, J .Neurosci 30:14972-14979, 2010.

2. H.F. Haider, T. Bojić, S.F. Ribeiro, J. Paço, D.A. Hall, A.J. Szczepek Pathophysiology of subjective tinnitus: triggers and maintenance, Front. Neurosci., vol. 12:866, 2018

3. W.A. Yost. Fundamentals of hearing, Academic Press, San Diego pp. 71-9,2000 
4. W. R. J. Funnell, N. Maftoon, and W. F. Decraemer. Modeling of middle ear mechanics, in The Middle Ear: Science, Otosurgery, and Technology (Springer, New York), pp. 171-210, 2013.

5. F. Zhao, T. Koike, J. Wang, H. Sienz, and R. Meredith. Finite element analysis of the middle ear transfer functions and related pathologies, Med. Eng. and Phys., vol. 31, pp. 907-916, 2009.

6. J. J. Rosowski, J. T. Cheng, M. E. Ravicz, N. Hulli, M. HernandezMontes, E. Harrington, and C. Furlong. Computer-assisted time-averaged holograms of the motion of the surface of the mammalian tympanic membrane with sound stimuli of 0.4-25 kHz, Hear Res. Vol. 253,pp. 83-96, 2009.

7. J. T. Cheng, M. Hamade, S. N. Merchant, J. J. Rosowski, E. Harrington, and C. Furlong. Wave motion on the surface of the human tympanic membrane: Holographic measurement and modeling analysis, J. Acoust. Soc. Am. Vol. 133(2), pp. 918-937, 2013.

8. S. Puria and C. Steele. Tympanic-membrane and malleus-incus-complex co-adaptations for high-frequency hearing in mammals, Hearing Res, vol. 263(1), pp. 183-190, 2010.

9. J. Zwislocki. Analysis of the middle ear function. part i. input impedance, J.Acoust. Soc. Am., vol. 34, 1962

10. M. E. Lutman and A. Martin. Development of an electroacoustic analogue model of the middle ear and acoustic reflex, J. Sound Vib., vol. 64, pp. 133-157, 1979.

11. M. Kringlebotn. Network model for the human middle ear, Scand Audiol 17:75-85, 1988.

12. J. Pascal, A. Bourgeade, M. Lagier, and C. Legros. Linear and nonlinear model of the human middle ear, J. Acoust. Soc. Am., vol. 104, no. 3, pp. 1509- 1516, 1998.

13. W. J. Song. Study on Human Auditory System Models and Risk Assessment of Noise Induced Hearing Loss, Ph.D. Dissertation, University of Cincinnati, February 2010.

14. J. J. Rosowski, M. E. Ravicz, and J. E. Songer. Structures that contribute to middle-ear admittance in chinchilla, J. Comp. Physiol. A:Neuroethol. Sens. Neural Behav. Physiol.vol. 192, pp. 1287-1311. 2006.

15. P. Bowers, and J. J. Rosowski. A lumped-element model of the chinchilla middle ear, J. Acoust. Soc. Am, vol. 145(4), pp.1975-1992, 2019.

16. K. N. O'Connor, and S. Puria, "Middle-ear circuit model parameters based on a population of human ears," J. Acoust. Soc. Am. 123, pp.197-21, 2008.

17. N. Verdier, A. Diet, and S. Megherbi. Matrices et quadripôle, une passerelle entre les mathématiques et l'électronique Matrices et quadripôles , une passerelle entre les mathématiques et l' électronique, J3eA 8, vol. 8, no. 1011, p. 8, 2009.

18. S. E. Voss, H.H. Nakajima, A.M. Huber, C.A. Shera. Function and acoustics of the normal and diseased middle ear, In: Puria S, Fay RR, Popper AN (eds) The middle ear. Springer, New York, pp 67-91, 2013

19. M. Riad, J. Bakkoury, O. Bouattane. Middle ear frequency response analysis for tinnitus identification. in proc. 2017 Int.conf on electrical and information technologies.10.1109/EITech.2017.8255228

20. S. E. Voss, G. R. Merchant, and N. J. Horton. Effects of middle-ear disorders on power reflectance measured in cadaveric ear canals, Ear. Hear, vol. 33, pp. 195-208, 2012.

21. S. E. Voss, J. J. Rosowski, S. N. Merchant, and W. T. Peake. Middle-ear function with tympanic-membrane perforations. II. A simple model, J. Acoust. Soc. Am,vol. 110,pp. 1445-145, 2001.

22. D. Lim. Structure and function of the tympanic membrane: A review, Acta otorhino-laryngologica Belg, vol. 49,pp. 101-115,1995

23. J. Kim, M. Koo. Mass and stiffness impact on the middle ear and the cochlear partition, J Audiol Otol, vol. 19, pp. 1-6, 2015.

24. V. Henriques et al. Abnormal Congenital Location of Stapes' Superstructure: Clinical and Embryological Implications, Case Rep. Otolaryngol., vol. 2016, no. Figure 1, pp. 1-4, 2016.

25. X. Zhang and R. Z. Gan. Finite element modeling of energy absorbance in normal and disordered human ears, Hear. Res., vol. 301, pp. 146-155, 2013.

26. S. B. Shende, A. B. Deoghare, and K. M. Pandey. Characterization of harmonic response of human middle ear using finite element approach, J. Comput. Sci., vol. 29, pp. 94-98, 2018.

27. O. Valentin. Auditory functions of the peripheral hearing system and the common conditions affecting sound conduction, Can. Acoust., vol. 47, no. June, 2019. 